\title{
Archéopages
}

Archéopages

Archéologie et société

\section{Les camps de prisonniers allemands. Un nouveau champ de recherche pour l'archéologie française}

\section{Vincent Carpentier et Cyril Marcigny}

\section{(2) OpenEdition}

1 Journals

Édition électronique

URL : https://journals.openedition.org/archeopages/549

DOI : 10.4000/archeopages.549

ISSN : 2269-9872

\section{Éditeur}

INRAP - Institut national de recherches archéologiques préventives

\section{Édition imprimée}

Date de publication : 1 septembre 2014

Pagination : 64-69

ISSN : $1622-8545$

\section{Référence électronique}

Vincent Carpentier et Cyril Marcigny, "Les camps de prisonniers allemands. Un nouveau champ de recherche pour l'archéologie française », Archéopages [En ligne], 39 | 10/2013-01/2014, mis en ligne le 01 janvier 2016, consulté le 21 janvier 2022. URL : http://journals.openedition.org/archeopages/549 ; DOI : https://doi.org/10.4000/archeopages.549 


\title{
Les camps de prisonniers allemands Un nouveau champ de recherche pour l'archéologie française
}

\author{
Vincent Carpentier Inrap, UMR 6273, Centre Michel de Boüard \\ Cyril Marcigny Inrap, UMR 6566-CReAAH
}

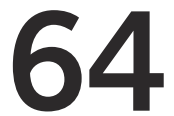

Au lendemain de la capitulation allemande, ratifiée le 8 mai 1945 à Karlshort, à l'est de Berlin, la France retient sur son sol un million de prisonniers dont $90 \%$ de nationalité allemande. Les trois quarts environ de ces hommes (740 ooo) ont été mis à disposition des autorités françaises par l'armée des États-Unis, à partir de février 1945 (Théofilakis, 2007, 2010 et 2014).

\section{De l'Allemagne à la France}

La plupart de ces anciens soldats avaient séjourné dans l'un des dix-neuf camps (Prisoners of War Temporary Enclosures [PWTE], ou Rheinwiesenlager) installés en Allemagne de l'ouest par les Américains dans la phase finale du conflit [ill. 1]. Ces créations venaient à la suite d'une décision prise dès le printemps 1943 par Eisenhower, visant à retirer à cette énorme masse de détenus le statut officiel de prisonniers de guerre au profit de celui de forces ennemies désarmées appartenant à un État qui avait cessé d'exister légalement. Il s'agissait, par ce moyen, de contourner les obligations imposées par la Convention de Genève vis-à-vis des prisonniers de guerre. En effet, lors de la percée finale vers Berlin, les autorités militaires alliées rencontrèrent de considérables difficultés logistiques pour faire face à quelque deux millions de prisonniers allemands. Après le franchissement du Rhin et la capture de Remagen, les Britanniques ne furent plus en mesure d'accueillir davantage de prisonniers, et abandonnèrent la tâche aux Êtats-Unis, qui durent improviser. La responsabilité s'avérait colossale : en mai 1945, les forces alliées avaient fait au total 3,4 millions de prisonniers des forces de l'Axe. Dès le mois d'avril, les Américains lancèrent en Allemagne de l'ouest la construction d'immenses camps de transit, vastes enclos ceints de barbelés, situés à l'écart des habitations, auxquels on accédait par le chemin de fer. La gestion interne du camp fut confiée à des personnels allemands.

D'avril à septembre 1945, les conditions de détention dans les PWTE furent extrêmement rudimentaires. Les maigres ressources de l'Allemagne en ruine étaient de très loin insuffisantes pour nourrir et soigner les prisonniers, et plusieurs milliers moururent de faim, de soif, de maladie ou d'épuisement. Dès le mois de mai 1945, les autorités américaines entreprirent de libérer certaines catégories de détenus. Les premiers furent des jeunes enrôlés dans les Jeunesses hitlériennes et des femmes sans lien direct avec le parti national-socialiste. Les travailleurs agricoles et de l'industrie, ouvriers, mécaniciens, mineurs, furent ensuite relâchés en vue de lancer les chantiers de reconstruction du pays. À la fin du mois de juin 1945, les camps de Remagen, de Böhl-Iggelheim et de Büderich fermèrent leurs portes après le départ de leurs derniers occupants. Le 12 juin, les autorités britanniques se virent confier la responsabilité de deux camps situés dans leur zone d'occupation en Allemagne de l'ouest (Büderich et Rheinberg). Bientôt, les Britanniques requirent pour les travaux de reconstruction les hommes valides placés sous leur contrôle, et libérèrent le reste des détenus. Puis, le 10 juillet, le haut-commandement américain décida de céder le contrôle des autres camps à la France. En effet, le nouveau gouvernement de Charles de Gaulle réclamait un contingent de 1,75 million de prisonniers au titre des réparations de guerre, afin de l'aider à reconstruire le pays : 182 ooo prisonniers furent donc livrés à la France, et vinrent rejoindre ceux déjà détenus. À la fin du mois de septembre 1945, dix-sept PWTE avaient été fermés. Seuls furent 


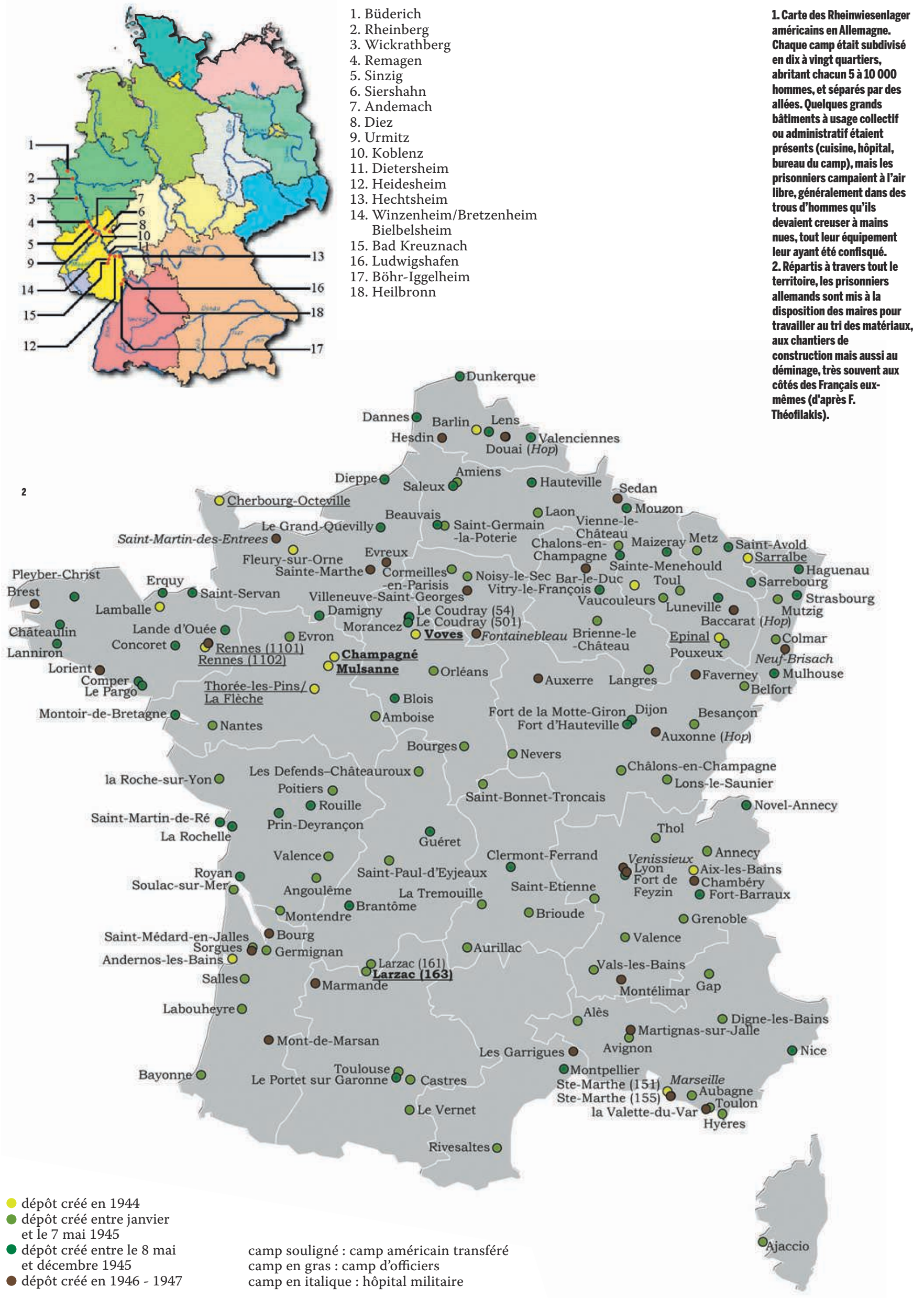


3. Nombre et répartition des prisonniers allemands en France entre 1945 et 1948.

La part des régions militaires

(RM, dont le découpage a

changé entre 1947 et 1948)

indique le pourcentage du

total des prisonniers

allemands présents sur le sol

français recensé dans telle

ou telle RM - les niveaux de

gris montrent les classes en

pourcentage et les cercles les

classes en valeur absolue.

Sur les 24178 prisonniers de

guerre officiellement décédés,

5745 furent victimes

des conditions de vie dans

les dépôts français.

Les campagnes de déminage

causèrent quant à elles

500 morts français et

5000 morts allemands

(d'après F. Théofilakis).

8
4. Au camp de la Glacerie (Manche), 180 cabanes semi-enterrées de $10 \mathrm{~m}^{2}$ environ étaient disposées en rangées ou " cages 》" (compounds) et chauffées au moyen de poêles

de fortune et des structures de chauffe collectives.

5. Cette vareuse d'un

prisonnier du camp de la

Glacerie (Manche) a été

retaillée à partir de toiles de

tente de l'armée américaine,

et marquée des lettres POW

- prisoner of war.

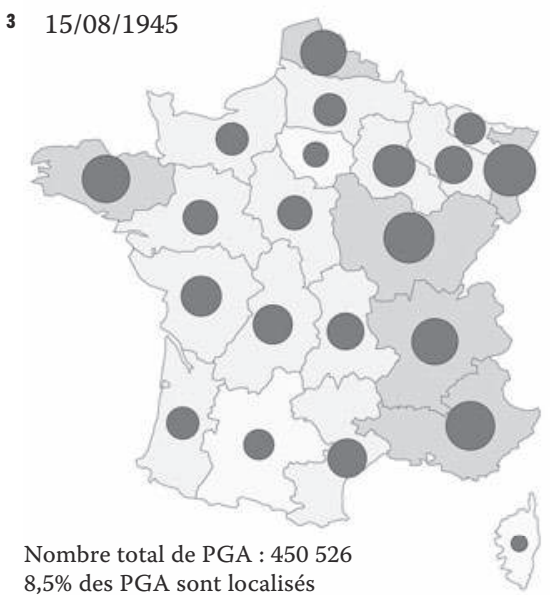

$8,5 \%$ des PGA sont localisés

dans la $10^{\mathrm{e}} \mathrm{RM}$ (Strasbourg)

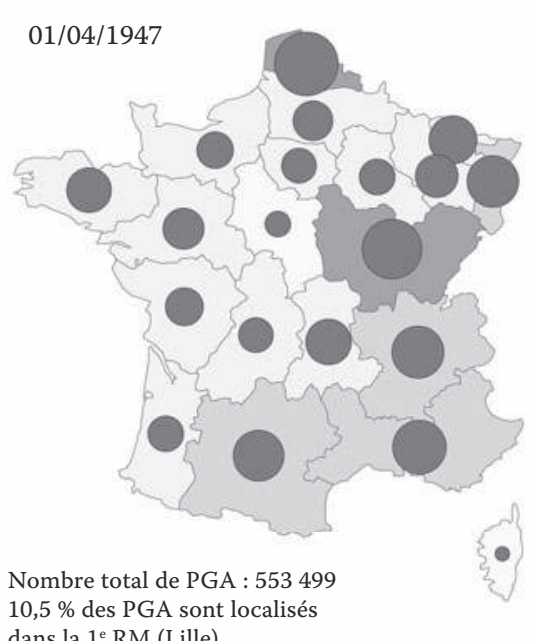

$10,5 \%$ des PGA sont localisé

ans la $1^{e} \mathrm{RM}$ (Lille)

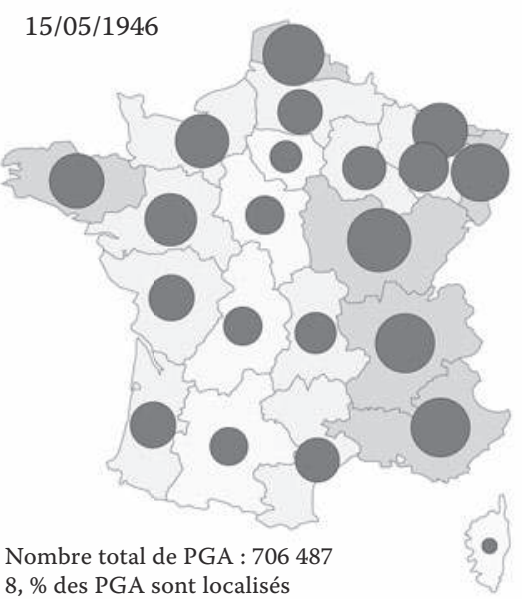

$\%$ des PGA sont localisés dans la $8^{\mathrm{e}} \mathrm{RM}$ (Dijon)

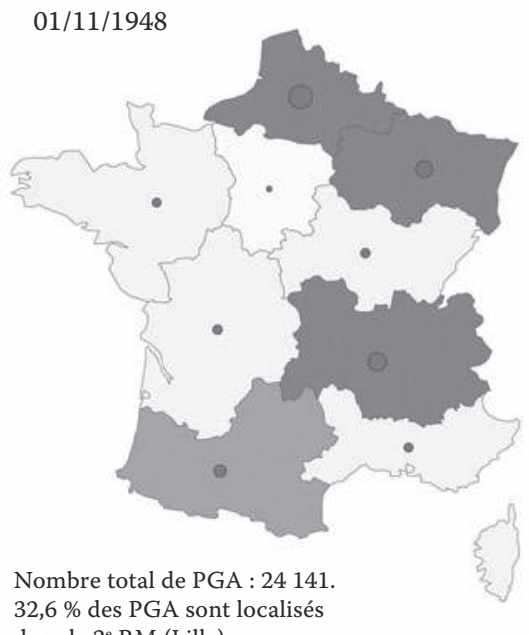

$32,6 \%$ des PGA son
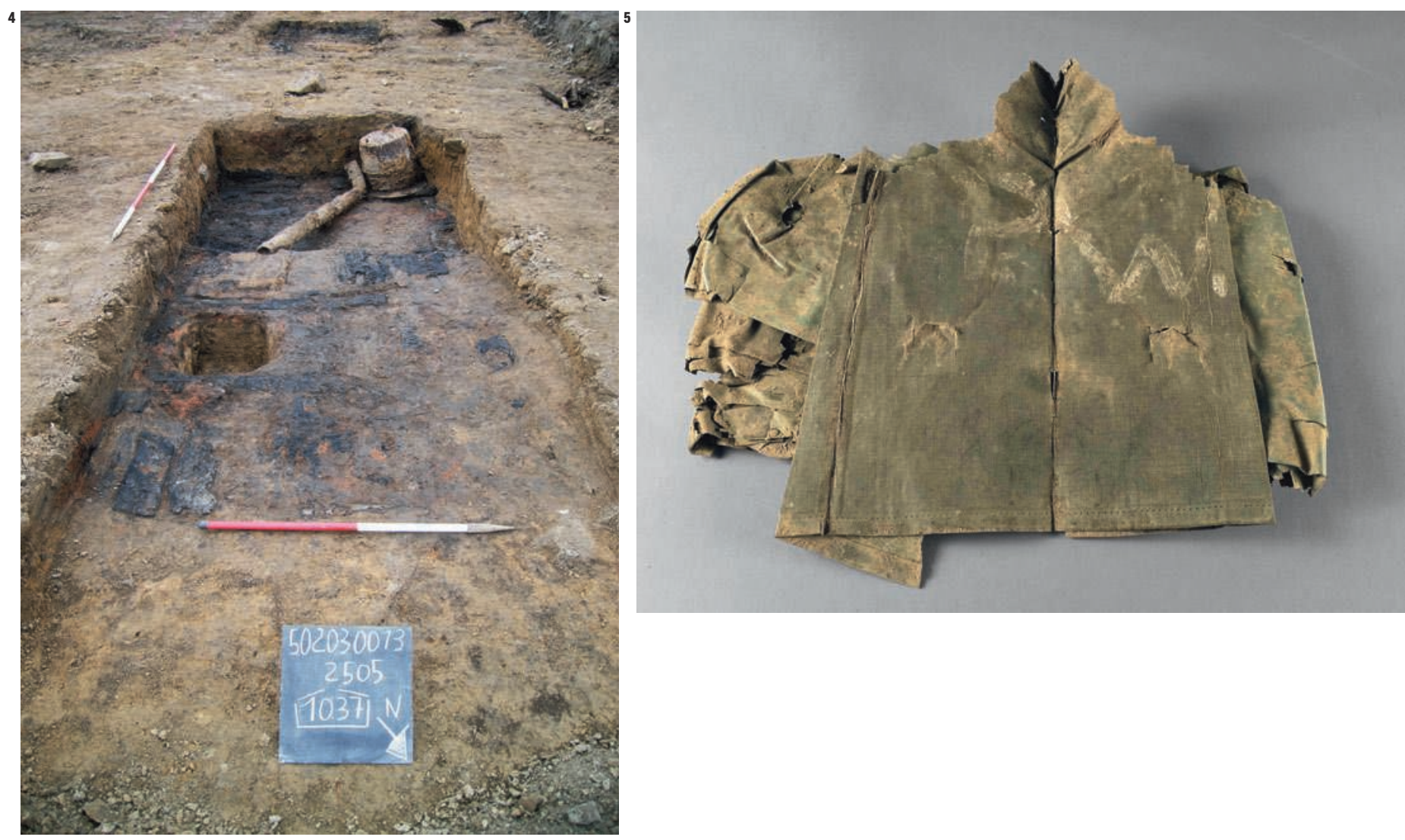

Part des RM:

Plus de $15 \%$

De 9 à $15 \%$

De 6 à $9 \%$

De 3 à $6 \%$

Nombre

de prisonniers :

58193

7873

- 2178

- 432 
maintenus jusqu'en 1948 les deux camps de Bretzenheim et Bad Kreuznach désormais utilisés comme camps de transit pour les prisonniers de retour de France.

Les ex-soldats allemands retenus en France furent envoyés dans des dizaines de camps disséminés à travers dans tout le pays [ill. 2], afin d'être employés à la Reconstruction, dans l'industrie et l'agriculture ainsi qu'au déminage, jusqu'en 1948 (Peschanski, 200o). Leur nombre atteint son maximum en 1946, avec plus de 700 ooo prisonniers [ill. 3]. La plupart furent rapatriés à partir d'avril 1947, les derniers à rester sur le sol français (soit 24, ooo hommes environ au $1^{\text {er }}$ novembre 1948) ayant opté pour un statut de travailleur civil libre.

\section{Prisonniers allemands en Normandie}

Au sortir de la Bataille de Normandie, fin août 1944, l'Allemagne laisse sur place 250 ooo prisonniers aux mains des Alliés, soit un quart du million de soldats de la Wehrmacht qui seront retenus captifs sur tout le territoire français (Quellien, 1992).

Depuis plusieurs années, Valentin Schneider, jeune historien bas-normand d'origine allemande, s'est penché sur ce sujet oublié, pour ne pas dire tabou, dans le cadre d'une thèse de doctorat dirigée par Jean Quellien, professeur émérite à l'université de Caen (Schneider, 2011). Ses travaux, qui ont nourri des discussions passionnées parmi les historiens, ont mis en lumière certaines modalités d'une cohabitation aujourd'hui encore mal assumée par la population française et en l'occurrence normande, bien qu'elle lui ait permis d'opérer son relèvement (Le Roc'h Morgère, 2011).

Les archives historiques révèlent qu'au lendemain même du 6 juin 1944, les premiers prisonniers allemands sont parqués par les Alliés dans des enclos de plage («beach enclosures ») [ill. de couverture] aménagés sur les dunes, au plus près des navires vers lesquels les premières files de détenus sont dirigées pour rejoindre des camps aménagés en Angleterre. Mais les capacités d'accueil de la Grande-Bretagne s'avèrent rapidement trop limitées, si bien qu'en août, les prisonniers sont désormais gardés en Normandie, où ils commencent à servir de réservoir de main-d'œuvre. Des camps sont alors ouverts sur place par les Alliés, au fil de leur progression, dans lesquels les détenus sont regroupés dans des conditions précaires, dans l'attente de connaître leur sort. Toutefois, même si leurs conditions de vie sont souvent très difficiles et que les vivres manquent, leur sort n'a rien à envier à celui de beaucoup de Français qui survivent alors dans les décombres de leur ville ou de leur village. En 1945, la totalité de ces camps passent sous autorité française, et de nouveaux contingents de détenus sont confiés par les États-Unis à la France, à charge de les nourrir, dans le but d'aider à la Reconstruction.

\section{Investigations récentes sur les camps de prisonniers}

Entre la fin des années 1990 et le début des années 2000, l'archéologie s'est pour la première fois investie sur ce sujet, à travers le diagnostic ou la fouille de plusieurs camps de prisonniers situés en Normandie (Carpentier, Marcigny, 2014). En 1998, une partie d'un camp britannique est étudiée à Mosles, près de Bayeux, à l'occasion de la fouille d'un site de l'âge du Fer ${ }^{\mathbf{1}}$. Des restes de fossés et de constructions sur poteaux sont dégagés, ainsi que divers objets dont un ensemble de fers de hachette, regroupés sans doute à l'écart des prisonniers par leurs gardiens. Les autres objets découverts consistent en ustensiles alliés et allemands, majoritairement liés à la vie quotidienne des prisonniers, tels que brosses à dents, rasoirs, lampes, outillage, ainsi que deux sommiers. Ce n'est guère toutefois avant l'année 2009 qu'une véritable problématique archéologique voit le jour autour de ce thème de recherche, lors de la fouille, menée par des archéologues anglais, d'un camp américain situé à La Glacerie, dans la Manche, à l'occasion de la construction d'un lotissement dans la banlieue de Cherbourg ${ }^{2}$ (Fichet de Clairfontaine, 2013). Les vestiges d'un camp de prisonniers allemands sont alors mis au jour [ill. 4] : l'importante collection de quelque 4 ooo objets consistant majoritairement en pièces d'habillement et équipement de l'armée américaine renseigne, là encore, le quotidien et les conditions de détention, et met en lumière les diverses solutions inventées par les prisonniers pour organiser leur survie (confection et réparation, troc alimentaire, hygiène et soins corporels, écriture...) [ill. 5]. Par ailleurs, ces vestiges sont mis en rapport avec les nombreux témoignages oraux et écrits concernant l'histoire de ce camp. L'ensemble débouche ainsi sur une exceptionnelle enquête interdisciplinaire, concernant un sujet à peu près totalement inédit comportant de fortes résonnances historiques mais aussi psychologiques. Ce site est désormais suivi régulièrement à l'occasion de diagnostics archéologiques ${ }^{3}$ permettant de compléter notre vision du camp et de ses espaces connexes.

Cette première expérience a depuis fait florès et, de 2009 à 2013, trois autres camps ont été partiellement investigués en Basse-Normandie. À Nonant-le-Pin, dans l'Orne, quelques fosses à usage de dépotoir, situées à proximité du camp proprement dit, ont été fouillées ${ }^{\mathbf{4}}$ [ill. 6]. À UrvilleNacqueville, dans la Manche, des sondages ont été ouverts en divers endroits d'un autre camp, permettant d'en évaluer l'étendue et d'en reconnaître les installations ${ }^{\mathbf{5}}$. Enfin, à Fleury-surOrne, dans le Calvados, c'est une portion du camp de la Grâce de Dieu qui devrait être prochainement explorée lors d'une vaste fouille concernant une vingtaine d'hectares ${ }^{\mathbf{6}}$ [ill. 7 et 8]. Ce camp de prisonniers britannique (Continental Central Prisoners of War Enclosures $n^{\circ} 2$ 221), fut rétrocédé à la France en 1945. Il s'agissait de l'un des camps 

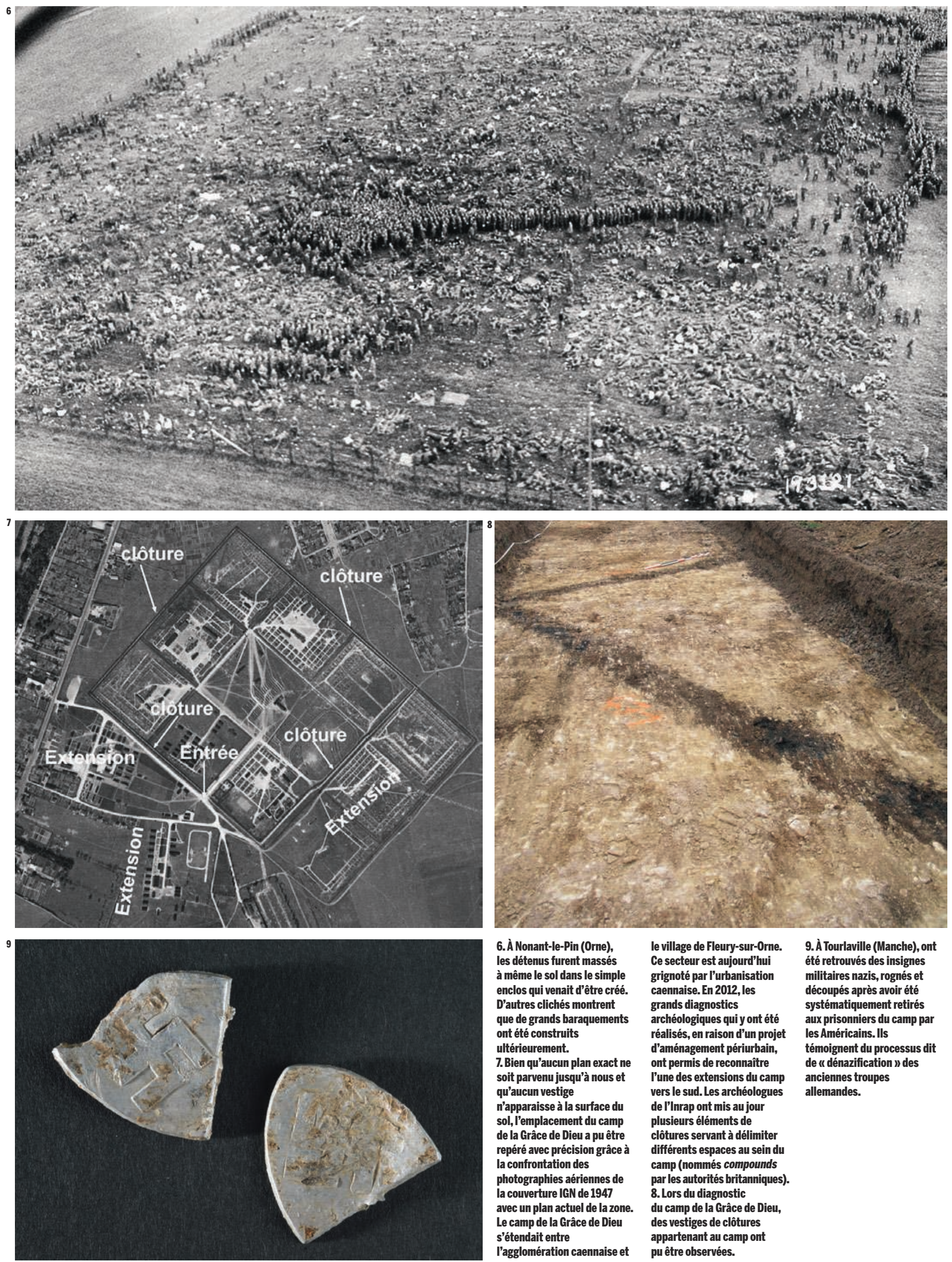

6. À Nonant-le-Pin (Orne), les détenus furent massés à même le sol dans le simple enclos qui venait d'être créé.

D'autres clichés montrent

que de grands baraquements ont été construits

ultérieurement.

7. Bien qu'aucun plan exact ne soit parvenu jusqu'à nous et qu'aucun vestige

n'apparaisse ala sol, l'emplacement du camp de la Grâce de Dieu a pu être repéré avec précision grâce à la confrontation des photographies aériennes de la couverture IGN de 1947 avec un plan actuel de la zone. Le camp de la Grâce de Dieu s'étendait entre

l'agglomération caennaise et le village de Fleury-sur-Orne. Ce secteur est aujourd'hui grignoté par I'urbanisation caennaise. En 2012, les

grands diagnostics

archéologiques qui y ont été réalisés, en raison d'un proje d'aménagement périurbain, ont permis de reconnaître l'une des extensions du camp vers le sud. Les archéologues de l'Inrap ont mis au jour plusieurs éléments de clôtures servant à délimiter différents espaces au sein du camp (nommés compounds par les autorités britanniques). 8. Lors du diagnostic

du camp de la Grâce de Dieu, des vestiges de clôtures appartenant au camp ont pu être observées.
9. À Tourlaville (Manche), ont été retrouvés des insignes militaires nazis, rognés et découpés après avoir été systématiquement retirés aux prisonniers du camp par les Américains. IIs témoignent du processus dit de «d dénazification ॥ des anciennes troupes allemandes. 
les plus importants du Calvados. Au plus fort de son activité, en 1946, il accueillit près de 12000 prisonniers allemands, employés notamment au déminage de la « cote 112 », hautlieu des combats entre les troupes allemandes et anglo-canadiennes, de juin à juillet 1944, au sud-ouest de Caen. Ces hommes étaient cantonnés sur un espace de près d'une trentaine d'hectares. Aucune trace du camp ne subsiste aujourd'hui en surface, mais le site, menacé de destruction par des travaux d'aménagement projetés, donnera lieu à une fouille au printemps 2014. Les objectifs scientifiques, d'ores et déjà fixés en amont de l'opération archéologique, portent sur plusieurs axes de recherche. Il s'agira tout d'abord de dresser le plan du camp et d'en saisir les différentes évolutions, depuis sa création en juillet 1944 jusquà sa fermeture, à la fin de l'année 1947. La fouille permettra aussi d'étudier précisément les modalités d'occupation des lieux, et de restituer la vie quotidienne des prisonniers à travers le prisme des nombreux objets laissés sur place. À terme, ces résultats seront confrontés aux sources écrites, iconographiques et ethnographiques.

Beaucoup d'autres camps de prisonniers identifiés en Normandie n'ont pas encore fait l'objet de véritables recherches archéologiques. C'est le cas notamment du camp de travail de Tourlaville, dans la Manche, à l'emplacement duquel ont été mis au jour, à la suite de travaux agricoles, de nombreux vestiges incluant des pièces de jeu ou des insignes métalliques portant la croix gammée qui ont été martelés, rognés ou découpés [ill. 9]. L'ensemble de ces données récentes et inédites mises au jour par l'archéologie recoupent, complètent et alimentent le travail de l'historien, dans le cadre d'une problématique renouvelée à l'échelle nationale.

Références bibliographiques

Carpentier V., Marcigny C., 2014, Archéologie du débarquement et de la bataille de Normandie, Rennes, Inrap/Ouest-France éditions, 144 p.

Fichet de Clairfontaine F., 2013, Le camp de travail de prisonniers de guerre allemands $112 A$, Archéologie en Basse-Normandie, n 2, Caen, Drac Basse-Normandie.

Le Roc'H Morgère L. (éd.), 2001, De la fin des camps à la Reconstruction. Les Normands, 1945-1947 (colloque international), Caen, Conseil général du Calvados, Direction des Archives départementales, $367 \mathrm{p}$.

PeSCHANSKI D., 2000, Les camps francais d'internement (1938-1946), Thèse de Doctorat d'Histoire sous la direction d'Antoine Prost, Université Paris I Panthéon-Sorbonne.

Quellien J., 1992, La Normandie au cour de la guerre, Rennes, Éditions Ouest-France, 247 p.

SCHNEIDER V., 2011, Un million de prisonniers allemands en France, 1944-1948, Paris, Éditions Vendémiaire, $191 \mathrm{p}$.

ThéOfILAKIS F., 2007, « Les prisonniers de guerre allemands en mains françaises dans les mémoires nationales en France et en Allemagne après 1945 ", Cahiers d'histoire. Revue d'histoire critique, 100, p. 67-84.

THÉOFILAKIS F., 2010, Les prisonniers de guerre allemands en mains françaises (1944-194.9): captivité en France, rapatriement en Allemagne, Thèse de Doctorat d'Histoire, sous la direction d'Annette Becker, Henry Rousso et Andreas Wirching, Université de Paris Ouest Nanterre la Défense. http://bdr.u-paris1o.fr/theses/ internet/2010PA100184_diff.pdf

ThÉOfILAKIS F., 2014, Les prisonniers de guerre allemands. France, 1944-1949, Paris, Fayard, 800 p. 